PROCEEDINGS OF THE

AMERICAN MATHEMATICAL SOCIETY

Volume 136, Number 7, July 2008, Pages 2625-2630

S 0002-9939(08)09387-8

Article electronically published on March 10, 2008

\title{
GEODESIC IDEAL TRIANGULATIONS EXIST VIRTUALLY
}

\author{
FENG LUO, SAUL SCHLEIMER, AND STEPHAN TILLMANN
}

(Communicated by Daniel Ruberman)

\begin{abstract}
It is shown that every non-compact hyperbolic manifold of finite volume has a finite cover admitting a geodesic ideal triangulation. Also, every hyperbolic manifold of finite volume with non-empty, totally geodesic boundary has a finite regular cover which has a geodesic partially truncated triangulation. The proofs use an extension of a result due to Long and Niblo concerning the separability of peripheral subgroups.
\end{abstract}

Epstein and Penner 2 used a convex hull construction in Lorentzian space to show that every non-compact hyperbolic manifold of finite volume has a canonical subdivision into convex geodesic polyhedra all of whose vertices lie on the sphere at infinity of hyperbolic space. In general, one cannot expect to further subdivide these polyhedra into ideal geodesic simplices such that the result is an ideal triangulation. That this is possible after lifting the cell decomposition to an appropriate finite cover is the first main result of this paper. A cell decomposition of a hyperbolic $n^{-}$ manifold into ideal geodesic $n$-simplices all of which are embedded will be referred to as an embedded geodesic ideal triangulation.

Theorem 1. Any non-compact hyperbolic manifold of finite volume has a finite regular cover which admits an embedded geodesic ideal triangulation.

The study of geodesic ideal triangulations of hyperbolic 3-manifolds goes back to Thurston [13. They are known to have nice properties through, for instance, work by Neumann and Zagier [10] and Choi [1. Petronio and Porti [1] discuss the question of whether every non-compact hyperbolic 3-manifold of finite volume has a geodesic ideal triangulation. This question still remains unanswered.

Kojima [6] extended the construction by Epstein and Penner to obtain a canonical decomposition into partially truncated polyhedra of any hyperbolic manifold with totally geodesic boundary components. A cell decomposition of a hyperbolic $n$-manifold with totally geodesic boundary into geodesic partially truncated $n^{-}$ simplices all of which are embedded will be referred to as an embedded geodesic partially truncated triangulation.

Received by the editors April 9, 2007.

2000 Mathematics Subject Classification. Primary 57N10, 57N15; Secondary 20H10, 22E40, $51 \mathrm{M} 10$.

Key words and phrases. Hyperbolic manifold, ideal triangulation, partially truncated triangulation, subgroup separability.

The research of the first author was supported in part by the NSF.

The second author was partly supported by the NSF (DMS-0508971).

The third author was supported under the Australian Research Council's Discovery funding scheme (project number DP0664276).

This work is in the public domain. 
Theorem 2. Any finite-volume hyperbolic manifold with non-empty, totally geodesic boundary has a finite regular cover which admits an embedded geodesic partially truncated triangulation.

An ideal polyhedron will be viewed as a special instance of a partially truncated one, which allows a unified proof of Theorems 1 1 and 2. They are proved by showing that any cell decomposition lifts to some finite regular cover where it can be subdivided consistently. In particular, one has the following application. Kojima [5] shows that any 3-dimensional finite-volume hyperbolic manifold with non-empty, totally geodesic boundary has a decomposition into geodesic partially truncated polyhedra each of which has at most one ideal vertex. Frigerio [3] conjectures that such a decomposition exists where each polyhedron is a tetrahedron; a virtually affirmative answer is an immediate consequence of the proof of Theorem 2 .

Corollary 3. Any 3-dimensional finite-volume hyperbolic manifold with non-empty, totally geodesic boundary has a finite regular cover which admits a decomposition into partially truncated geodesic tetrahedra each of which has at most one ideal vertex.

The key result used in the proof of Theorem 2 is the following theorem, which follows easily from work by Long and Niblo [8]. A subgroup $H$ of a group $G$ is separable in $G$ if given any element $\gamma \in G \backslash H$, there is a finite index subgroup $K \leq G$ that contains $H$ but $g \notin K$. If $M$ is a hyperbolic manifold of finite volume with (possibly empty) totally geodesic boundary, then a subgroup of $\pi_{1}(M)$ is termed peripheral if it is either conjugate to the fundamental group of a totally geodesic boundary component or to the fundamental group of a cusp or $\partial$-cusp.

Theorem 4 (Long-Niblo). Let $M$ be a hyperbolic manifold of finite volume with (possibly empty) totally geodesic boundary. Then every peripheral subgroup of $M$ is separable in $\pi_{1}(M)$.

\section{Subgroup Separability}

Let $M$ be a finite-volume hyperbolic $n$-manifold with non-empty totally geodesic boundary. Following Kojima [6], the periphery of $M$ is made up of three parts: first, $\partial M$ consisting of totally geodesic closed or non-compact hyperbolic $(n-1)$-manifolds; second, (internal) cusps modelled on closed Euclidean $(n-1)-$ manifolds; and third, $\partial$-cusps modelled on compact Euclidean $(n-1)$-manifolds with geodesic boundary. The boundary of $\partial$-cusps is contained on non-compact geodesic boundary components.

For the remainder of this paper, $M$ denotes a finite-volume hyperbolic manifold which is either non-compact or has non-empty totally geodesic boundary. Without loss of generality, it may be assumed that $M$ is orientable. Note that either $\widetilde{M}=\mathbb{H}^{n}$ or it can be viewed as the complement of an infinite set of hyperplanes in $\mathbb{H}^{n}$; in either case there is an identification $\pi_{1}(M)=\Gamma \leq \operatorname{Isom}^{+}\left(\mathbb{H}^{n}\right)$.

Proposition 5 (Long-Niblo). Let $X$ be a totally geodesic component of $\partial M$. Choose a basepoint $x \in X$. Then $\pi_{1}(X, x)$ is a separable subgroup of $\pi_{1}(M, x)$.

Proof. Let $D$ denote the manifold obtained by doubling $M$ along $X$. Then $D$ is hyperbolic with (possibly empty) totally geodesic boundary, and hence $\pi_{1}(D) \leq$ Isom $^{+}\left(\mathbb{H}^{n}\right)$ is residually finite due to a result by Mal'cev 9 . The proof in $\S 2$ of Long and Niblo [8] now applies to this setup. 
Proposition 6. Let $X$ be a horospherical cross section of a cusp or $\partial-$ cusp of $M$. Choose a basepoint $x \in X$. Then $\pi_{1}(X, x)$ is a separable subgroup of $\pi_{1}(M, x)$.

Proof. This follows from the well-known result that a maximal abelian subgroup of a residually finite group $\Gamma$ is separable in $\Gamma$ (see Ratcliffe [12]).

Proof of Theorem 4 . First note that if $\varphi: G_{1} \rightarrow G_{2}$ is an isomorphism and $H \leq$ $G_{1}$ is separable in $G_{1}$, then $\varphi(H) \leq G_{2}$ is separable in $G_{2}$. In particular, the reference to basepoints can be omitted. Next, note that if $H \leq \Gamma$ is separable, so is $\gamma^{-1} H \gamma$ for any $\gamma \in \Gamma$. Thus, Theorem 4 follows from the above propositions for orientable manifolds. If $M$ is non-orientable, denote by $\Gamma_{0}$ a subgroup of index two of $\Gamma$ corresponding to the fundamental group of the orientable double cover. Then any subgroup $H \leq \Gamma$ is separable in $\Gamma$ if and only if $H \cap \Gamma_{0}$ is separable in $\Gamma_{0}$. Now if $H$ is a peripheral subgroup of $\Gamma$, then $H \cap \Gamma_{0}$ is a peripheral subgroup of $\Gamma_{0}$.

\section{Partially truncated POlyhedra}

Certain convex geodesic polyhedra in $\mathbb{H}^{n}$ are termed geodesic partially truncated polyhedra and can be described intrinsically. However, reference to the projective ball model $B^{n} \subset \mathbb{R} P^{n}$ will be made here, and $\mathbb{H}^{n}$ will be identified with $B^{n}$ throughout.

Let $\hat{P}$ be an $n$-dimensional convex affine polyhedron in $\mathbb{R} P^{n}$ such that (1) each vertex is either called ideal or hyperideal, (2) its ideal vertices are contained on $\partial B^{n}$, (3) its hyperideal vertices are contained in $\mathbb{R} P^{n} \backslash \bar{B}^{n}$, and (4) each face of codimension two meets $\bar{B}^{n}$. Then a convex geodesic polyhedron $P \subset B^{n}$ is obtained by truncating $\hat{P}$ along hyperplanes canonically associated to its hyperideal vertices as follows. If $v \in \mathbb{R} P^{n}$ is a hyperideal vertex, then the associated hyperplane $H(v)$ is the hyperplane parallel to the orthogonal complement of $v$ which meets $\partial B^{n}$ in the set of all points $x$ with the property that there is a tangent line to $\partial B^{n}$ passing through $x$ and $v$. The polyhedron $P$ is termed a geodesic partially truncated polyhedron, and $\hat{P}$ the affine fellow of $P$. Combinatorially, $P$ is obtained from $\hat{P}$ by removing disjoint open stars of all the hyperideal vertices as well as all the ideal vertices.

If a codimension-one face of $P$ is contained in a face of $\hat{P}$, then it is called lateral; otherwise it is a truncation face. Lateral faces and truncation faces meet at right angles. If $P$ has no truncation faces, then it is also termed a geodesic ideal polyhedron.

Any subdivision of $\hat{P}$ into $n$-simplices without introducing new vertices uniquely determines a subdivision of $P$ into geodesic partially truncated $n$-simplices.

\section{The PUlLing CONSTRUCTION}

Let $(\mathcal{C}, \Phi)$ be a geodesic partially truncated cell decomposition of $M$, that is, $\mathcal{C}$ is a disjoint union of geodesic partially truncated polyhedra, each element in $\Phi$ is an isometric face pairing, and $M=\mathcal{C} / \Phi$. Then $(\mathcal{C}, \Phi)$ pulls back to a $\Gamma$-equivariant cell decomposition of $\widetilde{M} \subseteq B^{n}$, and for each $P \in \mathcal{C}$ one may choose an isometric lift $\tilde{P}$ to $B^{n}$ and hence an affine fellow $\hat{P} \subset \mathbb{R} P^{n}$. The hyperideal vertices of $\hat{P}$ correspond to totally geodesic boundary components of $M$, the ideal vertices of $\hat{P}$ to internal cusps, and the intersection of codimension-two faces of $\hat{P}$ with $\partial B^{n}$ to $\partial$-cusps. 
Let $\hat{\mathcal{C}}=\bigcup\{\hat{P}\}$ be the finite disjoint union of the affine fellows, and view $P \subset \hat{P}$. The cell decomposition of $M$ induces face pairings $\hat{\Phi}$ such that $M$ is obtained from the pseudo-manifold $\hat{M}=\hat{\mathcal{C}} / \hat{\Phi}$ by deleting the ideal vertices and open stars of the hyperideal vertices, and each element of $\hat{\Phi}$ restricts to an element of $\Phi$.

The following subdivision procedure is akin to Lemma 1.4 in Hudson 4] and constructions found in Section 17.2 of Lee [7].

Lemma 7 (Pulling construction). Suppose that no polyhedron in $\hat{\mathcal{C}}$ has two distinct vertices identified in $\hat{M}$. Then $M$ has an embedded geodesic partially truncated triangulation.

Proof. It suffices to show that there is a subdivision of $(\hat{\mathcal{C}}, \hat{\Phi})$ such that (1) each polyhedron in $\hat{\mathcal{C}}$ is simplicially subdivided into straight affine $n$-simplices without introducing new vertices, and (2) the elements of $\hat{\Phi}$ restrict to simplicial face pairings with respect to the subdivision.

Choose an ordering of the cusps and totally geodesic boundary components of $M$. This determines a well-defined, unique ordering of the 0 -skeleton of $\hat{M}$ and, by assumption, of the vertices of each polyhedron in $\hat{\mathcal{C}}$. One thus obtains the following unique subdivision of each polyhedron.

Let $P \in \mathcal{C}$, and label its vertices $v_{0}, v_{1}, \ldots, v_{k}$ such that $v_{i}>v_{j}$ if $i<j$. Subdivide $\hat{P}$ by coning to $v_{0}$ each element of its $i$-skeleton, $0 \leq i \leq n-1$, that does not contain $v_{0}$. The result is a collection of polyhedra, $\mathcal{P}_{0}$, together with well-defined face pairings $\Phi_{0}$ such that the identification space $\mathcal{P}_{0} / \Phi_{0}$ is $\hat{P}$. One now proceeds inductively. Given $\mathcal{P}_{j}$ and $\Phi_{j}$, subdivide each polyhedron in $\mathcal{P}_{j}$ containing $v_{j+1}$ by coning to $v_{j+1}$ each element of its $i$-skeleton, $0 \leq i \leq n-1$, that does not contain $v_{j+1}$. This gives $\mathcal{P}_{j+1}$, together with well-defined face pairings $\Phi_{j+1}$ such that the identification space $\mathcal{P}_{j+1} / \Phi_{j+1}$ is $\hat{P}$.

It needs to be shown that the set $\mathcal{P}_{k}$ is a collection of $n$-simplices. Indeed, let $Q \in \mathcal{P}_{k}$, and assume that $v_{h}$ is its smallest vertex. Then $Q$ is the cone to $v_{h}$ of an $(n-1)$-dimensional face $F^{n-1}$ not containing $v_{h}$. The face $F^{n-1}$ is the cone to its smallest vertex of an $(n-2)$-dimensional face $F^{n-2}$ not containing that vertex, and it follows inductively that $Q$ has exactly $n+1$ vertices.

Let $\hat{P}, \hat{P}^{\prime} \in \hat{\mathcal{C}}$ with top-dimensional faces $\hat{F}, \hat{F}^{\prime}$ such that there is a face pairing $\varphi \in \hat{\Phi}$ with $\varphi(\hat{F})=\hat{F}^{\prime}$. The respective subdivisions of $\hat{F}$ and $\hat{F}^{\prime}$ into ideal $(n-1)-$ simplices depend uniquely on the ordering of their vertices, whence $\varphi$ is simplicial with respect to the subdivisions, and restricts to a simplicial face pairing for each $n^{-}$ simplex in the subdivision. Moreover, the resulting decomposition of $\hat{M}$ is simplicial since any $n$-simplex has no two vertices identified, and hence must be embedded in $\hat{M}$.

\section{Proof of the main Results}

The strategy of the proof is to create a finite regular cover $N$ of $M$ with the property that Lemma 7 can be applied to the pull-back of $\mathcal{C}$. The notation of the previous sections will be used. Recall that for each $P \in \mathcal{C}$, there is the fixed affine fellow $\hat{P} \subset \mathbb{R} P^{n}$. The action of $\Gamma$ on $\bar{B}^{n}$ extends to $\mathbb{R} P^{n} \backslash \bar{B}^{n}$ via the action on the associated hyperplanes. In particular, if $v \in \hat{P}$ is a vertex, then the subgroup $\operatorname{Stab}_{\Gamma}(v) \leq \Gamma$ is peripheral. 
Let $\mathcal{D}(M)$ be the following set of pairs of points in $\mathbb{R} P^{n}:(v, w) \in \mathcal{D}(M)$ if and only if there is some $P \in \mathcal{C}$ such that $v$ and $w$ are distinct vertices of $\hat{P}$. Note that $\mathcal{D}(M)$ is finite; its elements are termed diagonals for $M$. A diagonal $(v, w)$ is said to be returning if there is $\gamma \in \Gamma$ such that $\gamma v=w$. Note that the pulling construction can be applied to $\hat{\mathcal{C}}$ if no diagonal is returning. Hence assume that this is not the case.

If $p: N \rightarrow M$ is a finite cover, then the cell decomposition $(\mathcal{C}, \Phi)$ pulls back to a cell decomposition of $N$, and there is a corresponding set of diagonals for $N$. If $P \in \mathcal{C}$ pulls back to $P_{1}, \ldots, P_{k}$, then (up to relabelling) one may choose $\hat{P}=\hat{P}_{1}$. In particular, it may be assumed that $\mathcal{D}(M) \subset \mathcal{D}(N)$; any other element of $\mathcal{D}(N)$ is of the form $\gamma \cdot(v, w)=(\gamma v, \gamma w)$ for some $\gamma \in \Gamma$ and $(v, w) \in \mathcal{D}(M)$. This choice will be made throughout. If $(v, w) \in \mathcal{D}(M)$ is not a returning diagonal for $M$, then it is also not a returning diagonal for $N$.

Assume that $(v, w) \in \mathcal{D}(M)$ is a returning diagonal.

Lemma 8. There is a finite (possibly not regular) cover $p: N_{(v, w)} \rightarrow M$ such that $(v, w) \in \mathcal{D}(N)$ is not a returning diagonal.

Proof. Since $(v, w)$ is a returning diagonal for $M$, there is $\gamma \in \Gamma$ such that $\gamma v=w$.

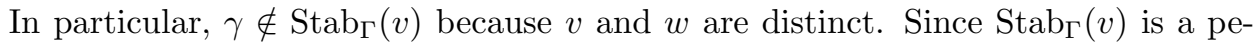
ripheral subgroup, Theorem 4 yields a finite index subgroup $K \leq \Gamma$ which contains $\operatorname{Stab}_{\Gamma}(v)$ but $\gamma \notin K$. Denote by $p: N_{(v, w)} \rightarrow M$ the finite cover corresponding to the subgroup $K$, i.e. $N_{(v, w)}=\widetilde{M} / K$.

Assume that $(v, w) \in \mathcal{D}(N)$ is a returning diagonal. Then there is $\delta \in K$ with the property that $\delta v=w$. Thus, $\gamma^{-1} \delta \in \operatorname{Stab}_{\Gamma}(v) \leq K$, which implies $\gamma \in K$. But this contradicts the choice of $K$, whence $(v, w)$ is not a returning diagonal for $N$.

Lemma 9. If $N \rightarrow M$ is a regular cover that factors through $N_{(v, w)}$, then no element of the orbit $\Gamma \cdot(v, w)$ can be a returning diagonal for $N$.

Proof. If $N \rightarrow N_{(v, w)} \rightarrow M$, where $N \rightarrow M$ is a regular cover, then $(v, w)$ cannot be a returning diagonal for $N$. If $N \rightarrow M$ is a regular cover, then no element of the orbit $\Gamma \cdot(v, w)$ is a returning diagonal for $N$ since the action of the group of deck transformations is transitive and $\pi_{1}(N)$ corresponds to a normal subgroup of $\pi_{1}(M)$.

For each diagonal $(v, w)$ choose a finite cover $N_{(v, w)} \rightarrow M$ with the property that $(v, w)$ is not a returning diagonal for $N_{(v, w)}$. This gives a finite collection of covers, and one may pass to a common finite cover $N$ with the property that $N \rightarrow M$ is regular. In particular, no element of the orbit of any diagonal for $M$ can be a returning diagonal for $N$. The cell decomposition $(\mathcal{C}, \Phi)$ of $M$ lifts to a polyhedral cell decomposition of $N$, to which the pulling construction can thus be applied. This completes the proof of Theorems 1 and 2 ,

\section{ACKNOWLEDGMENTS}

The authors thank Chris Leininger for an enlightening conversation. They also thank Daryl Cooper for bringing the work by Long and Niblo to their attention. 


\section{REFERENCES}

1. Young-Eun Choi: Positively oriented ideal triangulations on hyperbolic three-manifolds, Topology 43, no. 6, 1345-1371 (2004). MR2081429 (2005i:57016)

2. David B. A. Epstein and Robert C. Penner: Euclidean decompositions of noncompact hyperbolic manifolds, J. Differential Geom. 27, no. 1, 67-80 (1988). MR918457 (89a:57020)

3. Roberto Frigerio: On deformations of hyperbolic 3-manifolds with geodesic boundary, Algebr. Geom. Topol. 6, 435-457 (2006). MR2220684 (2007b:57027)

4. John Hudson: Piecewise linear topology, University of Chicago Lecture Notes, W. A. Benjamin, Inc., New York-Amsterdam, 1969. MR0248844 (40:2094)

5. Sadayoshi Kojima: Polyhedral decomposition of hyperbolic 3-manifolds with totally geodesic boundary. Aspects of low-dimensional manifolds, 93-112, Adv. Stud. Pure Math., 20, Kinokuniya, Tokyo, 1992. MR1208308 (94c:57023)

6. Sadayoshi Kojima: Polyhedral Decomposition of Hyperbolic Manifolds with Boundary, On the geometric structure of manifolds, edited by Dong Pyo Chi, Proc. of Workshop in Pure Math., 10, part III, 37-57 (1990).

7. Carl W. Lee: Subdivisions and triangulations of polytopes, Handbook of discrete and computational geometry, 271-290, CRC Press Ser. Discrete Math. Appl., CRC, Boca Raton, FL, 1997. MR:1730170

8. Darren D. Long and Graham A. Niblo: Subgroup separability and 3-manifold groups, Math. Z. 207, no. 2, 209-215 (1991). MR1109662 (92g:20047)

9. Anatoliı Ivanovich $\mathrm{Mal}^{\prime} \mathrm{cev}$ : On isomorphic matrix representations of infinite groups, Rec. Math. [Mat. Sbornik] N.S. 8 (50), 405-422 (1940). MR0003420 (2:216d)

10. Walter D. Neumann and Don Zagier: Volumes of hyperbolic three-manifolds, Topology 24, 307-332 (1985). MR815482 (87j:57008)

11. Carlo Petronio and Joan Porti: Negatively oriented ideal triangulations and a proof of Thurston's hyperbolic Dehn filling theorem, Expo. Math. 18, no. 1, 1-35 (2000). MR.1751141 (2001c:57017)

12. John G. Ratcliffe: Foundations of hyperbolic manifolds, Graduate Texts in Mathematics, 149, Springer-Verlag, New York, 1994. MR1299730 (95j:57011)

13. William P. Thurston: The geometry and topology of 3-manifolds, Princeton Univ. Math. Dept. (1978). Available from http://msri.org/publications/books/gt3m/.

Department of Mathematics, Rutgers University, New Brunswick, New Jersey 08854 E-mail address: fluo@math.rutgers.edu

Department of Mathematics, Rutgers University, New Brunswick, New Jersey 08854

E-mail address: saulsch@math.rutgers.edu

Department of Mathematics and Statistics, The University of Melbourne, ViC 3010, Australia

E-mail address: tillmann@ms.unimelb.edu.au 